Pacific Journal of Mathematics

CHARACTERISATIONS OF AMENABLE LOCALLY COMPACT 


\title{
CHARACTERISATIONS OF AMENABLE LOCALLY COMPACT SEMIGROUPS
}

\author{
JAMEs C. S. Wong AND A. Riazi
}

Let $G$ be a locally compact group, $\mathcal{S}=\mathscr{S}(G)$ the space of all (real) measurable simple functions on $G$ and $P=P(G)=\left\{\varphi \in L_{\mathrm{l}}(G): \varphi \geq 0\right.$, $\left.\|\varphi\|_{1}=1\right\}$. W. R. Emerson recently proved that the following conditions on $G$ are equivalent: (a) $G$ is amenable (i.e. $L_{\infty}(G)$ or $\delta$ has a left invariant mean; (b) $\varkappa_{P}(G)$ is closed under addition; (c) $d\left(\varphi_{1} * P, \varphi_{2} * P\right)=0$ for $\varphi_{1}, \varphi_{2} \in P$. Here $\Re_{P}(G)$ is the set of all $f \in \mathcal{S}$ such that $\inf \left\{\|\varphi * f\|_{\infty}: \varphi \in P\right\}=0$ and $d\left(\varphi_{1} * P, \varphi_{2} * P\right)=$ $\inf \left\{\left\|\varphi_{1} * \varphi-\varphi_{2} * \psi\right\|_{1}: \varphi, \psi \in P\right\}$. He also demonstrated that some well-known results and characterisations of amenability follow this as simple consequences. The main purpose of this paper is to show that similar (and more) results hold true for locally compact semigroups $S$ and invariant means on subspaces of $M(S)^{*}$, where $M(S)$ is the Banach algebra of all bounded regular Borel measures on $S$. We also provide an answer to a problem raised by Emerson. However, Emerson's arguments do not carry over in the absence of a Haar integral.

1. Introduction. Let $G$ be a locally compact group with group algebra $L_{1}(G), P=P(G)=\left\{\varphi \in L_{1}(G): \varphi \geq 0,\|\varphi\|_{1}=1\right\}$ and $\mathcal{S}=\mathcal{S}(G)$, the space of all (real) measurable simple functions on $G$. In [5] W. R. Emerson proved the following interesting results:

THEOREM (Emerson). The following conditions are equivalent:

(a) $G$ is amenable (i.e. $L_{\infty}(G)$ has a left invariant mean);

(b) $\Re_{P}(G)$ is closed under addition;

(c) $d\left(\varphi_{1} * P, \varphi_{2} * P\right)=0$ for any $\varphi_{1}, \varphi_{2} \in P$.

Here $d\left(\varphi_{1} * P, \varphi_{2} * P\right)=\inf \left\{\left\|\varphi_{1} * \varphi-\varphi_{2} * \psi\right\|_{1}, \varphi, \psi \in P\right\} \quad$ and $\Re_{P}(G)$ is the set of all functions $f \in \mathcal{S}$ such that inf $\left\{\|\varphi * f\|_{\infty}: \varphi \in P\right\}=0$ or, equivalently, 0 is in the norm (or weak) closure of the convex set $P * f$. Condition (a) is functional analytic in nature, (b) is algebraic and (c) is geometric. Emerson's arguments depend essentially on the Haar integral and the following:

Proposition (Emerson). There is a left invariant mean on $\mathcal{S}$ iff $\mathcal{S}$ contains a linear subspace $\Re$ satisfying the following conditions:

(i) $l_{a} f-f \in \mathcal{N}$ for all $f \in \mathcal{S}$ and $a \in G$;

(ii) $f \in \mathcal{S}$ and $f>0$ implies $f \notin \Re$, where $l_{a} f(x)=f(a x), x \in G$. 
This is basically the condition used by Banach [2] in 1923 to construct a left invariant mean for the circle group. The purpose of this paper is to obtain analogous results for a locally compact semigroup $S$ and invariant means on translation invariant linear subspaces of the dual $M(S)^{*}$ of the measure algebra $M(S)$. The absence of a Haar integral and the fact that we are dealing with functionals lead us to an interesting, but different, formulation and proof. Even in the special case of a group, our results are more general since we consider invariant means on spaces other than $L_{\infty}(G)$ or $\delta$ as well. We also provide an answer to a problem raised by Emerson [5, p. 187, after Proposition 1.8] (see $§ 4$ below).

For terminologies regarding invariant means on locally compact semigroups, the reader is referred to Wong [18]. Also, the present paper can be regarded as a sequel to that of Emerson [5], and we shall freely use their terminologies as well.

Let $S$ be a locally compact semigroup (in which multiplication is separately continuous, see also [21]) with convolution measure algebra $M(S)$. For $F \in M(S)^{*}$ with $\mu \in M(S)$, we define $l_{\mu} F=\mu \odot F$ by $\mu \odot$ $F(\nu)=F(\mu * \nu), \nu \in M(S)$. If $\mu=\varepsilon_{a}$, the Dirac measure at $a \in S$, we sometimes write $l_{a} F$ for $l_{\varepsilon_{a}} F$. Let $M_{0}(S)=\{\mu \in M(S): \mu \geq 0$ and $\|\mu\|=$ 1 ), and $M_{1}(S)$ be the subset of $M_{0}(S)$ consisting of all finite convex combinations of Dirac measures. If $X$ is a linear subspace of $M(S)^{*}$ containing the constant functional 1 , where $1(\mu)=\int 1 d \mu=\mu(S), \mu \in$ $M(S)$. We say that $X$ is left invariant [topological left invariant] if $l_{a}(X) \subset X$ for any $a \in S\left[l_{\mu}(X) \subset X\right.$ for any $\left.\mu \in M_{0}(S)\right]$. An element $M \in X^{*}$ is called a mean if

$$
\inf \left\{F(\mu): \mu \in M_{0}(S)\right\} \leq M(F) \leq \sup \left\{F(\mu): \mu \in M_{0}(S)\right\}
$$

for any $F \in X . M$ is left invariant [topological left invariant] if $M\left(l_{a} F\right)=$ $M(F)$ for any $F \in X, a \in S\left[M\left(l_{\mu} F\right)=M(F)\right.$ for any $F \in X, \mu \in$ $M_{0}(S)$ ]. Also, denote by $\mathfrak{T}_{0}(X)$ the set of functionals $F$ in $X$ such that $\inf \left\{\|\mu \odot F\|: \mu \in M_{0}(S)\right\}=0$, and by $\Re_{1}(X)$ those for which $\inf \left\{\|\mu \odot F\|: \mu \in M_{1}(S)\right\}=0$. We shall see in $\S 2$ that for certain subspaces $X$ (including $X=M(S)^{*}$ ) if $X$ has a topological left invariant mean, then $\Re_{0}(X)$ coincides with the set of all functionals $F$ in $X$ which are topological left almost congruent to zero. That is, $M(F)=0$ for any topological left invariant mean on $X$.

\section{Basic results.}

Proposition 2.1. Let $X$ be a topological left invariant [left invariant] linear subspace of $M(S)^{*}$ containing the constant functional 1 . Then $X$ has a 
topological left invariant mean [left invariant mean] iff $X$ contains a linear subspace $\mathcal{N}$ with the following properties:

(1) $l_{\mu} F-F \in \Re$ for all $F \in X, \mu \in M_{0}(S)$;

[(1') $l_{a} F-F \in \Re$ for all $F \in X, a \in S$; ]

(2) $F \in X$ and $\inf \left\{F(\mu): \mu \in M_{0}(S)\right\}>0$ implies $F \notin \Re$.

Remarks. This is an analogue of Proposition 1.1 in Emerson [5] for groups. However, this is a major difference in formulation here. Namely, Emerson considered only the measurable simple functions $\delta=\delta(G)$ on a group $G$ (which is amenable iff $\delta$, hence $L_{\infty}(G)$, has a left invariant mean) because his proof depends on the fact that for a simple function $f \in \mathcal{S}$, $f>0$ iff inf $f>0$. This is not true for $f \in L_{\infty}(G)$. For such functions, the condition $f>0$ of Proposition 1.1 in Emerson [5] must be replaced by ess inf $f>0$. This will be discussed in $\S 4$. For semigroups $S$ and functionals $F$ in $M(S)^{*}$, the correct replacement is, of course, $\inf \{F(\mu): \mu \in$ $M(S)\}>0$ as stated in Proposition 2.1.

Proof. The proof is similar to Emerson's and is based on Dixmier's criterion for amenability. If $X$ has a topological left invariant mean $M$, then $\Re=\operatorname{ker} M$ has properties (1) and (2). Conversely, if $\Re$ satisfies (1) and (2), then by (1), $\mathscr{T}$ must contain $\mathcal{K}_{l}$, the linear space generated by functionals of the form $l_{\mu} F-F, F \in X, \mu \in M_{0}(S)$. By (2), $H \in \mathcal{H}_{l}$ implies $\inf \{H(\mu): \mu \in M(S)\} \leq 0$. Hence $X$ has a topological left invariant mean by Dixmier's criterion (cf. Hewitt and Ross [8, Theorem 17.4]). For left invariance, the proof is the same.

TheOREM 2.2. $\Re_{0}(X)\left[\Re_{1}(X)\right]$ satisfies conditions $(1)$ and $(2)\left[\left(1^{\prime}\right)\right.$ and (2)] of Proposition 2.1 and is closed under scalar multiplication.

Proof. We shall prove only the assertion about $\Re_{0}(X)$. Let $F \in X$ and $\mu \in M_{0}(S)$. Define a sequence $\mu_{n}=\frac{1}{n} \sum_{k=1}^{n} \mu^{k}$, where $\mu^{k}=\mu * \cdots * \mu$ ( $k$ times), and let $F_{n}=\mu_{n} \odot(\mu \odot F-F)$.

Then

$$
\begin{aligned}
F_{n} & =\left(\mu * \mu_{n}\right) \odot F-\mu_{n} \odot F \\
& =\frac{1}{n}\left\{\sum_{k=1}^{n} \mu^{k+1} \odot F-\sum_{k=1}^{n} \mu^{k} \odot F\right\}=\frac{1}{n}\left(\mu^{n+1} \odot F-\mu \odot F\right) .
\end{aligned}
$$

Hence $\left\|F_{n}\right\| \leq(2 / n)\|F\| \rightarrow 0$ as $n \rightarrow \infty$ and $\mu \odot F-F \in \mathscr{T}_{0}(X)$. 
Next, suppose $F \in X$ and $\inf \left\{F(\mu): \mu \in M_{0}(S)\right\}=\varepsilon>0$. Then for any $\nu \in M_{0}(S)$, we have

$$
\begin{aligned}
\|\nu \odot F\| & =\sup \{|F(\nu * \mu)|: \mu \in M(S),\|\mu\|=1\} \\
& \geq \sup \left\{|F(\nu * \mu)|: \mu \in M_{0}(S)\right\} \\
& \geq \inf \left\{|F(\nu * \mu)|: \mu \in M_{0}(S)\right\} \\
& \geq \inf \left\{|F(\mu)|: \mu \in M_{0}(S)\right\} \\
& =\varepsilon>0,
\end{aligned}
$$

which shows that $\inf \left\{\|\nu \odot F\|: \nu \in M_{0}(S)\right\}>0$ or $F \notin \Re_{0}(X)$.

Clearly, $\Re_{0}(X)$ is closed under scalar multiplication. The assertion about $\Re_{1}(X)$ can be similarly proved by using the facts that $\Re_{1}(X) \subset$ $\Re_{0}(X)$ and that if $\mu=\varepsilon_{a}$ is a Dirac measure, then

$$
\mu_{n}=\frac{1}{n} \sum_{k=1}^{n} \mu^{k}=\frac{1}{n} \sum_{k=1}^{n} \varepsilon_{\left(a^{k}\right)} \in M_{1}(S) .
$$

Remarks. Theorem 2.2 is a (topological) analogue of Proposition 1.3 in Emerson [5] for locally compact groups. His proof depends on the Haar integral and therefore cannot be carried over to semigroups. In fact our arguments above provide an alternative proof of Emerson's result for groups without invoking the Haar integral.

THEOREM 2.3. $X$ has a topological left invariant mean [left invariant mean $]$ if $\Re_{0}(X)\left[\Re_{1}(X)\right]$ is closed under addition.

\section{Proof. By Proposition 2.}

Next, we shall establish a condition for $\Re_{0}(X)$ and $\Re_{1}(X)$ to be closed under addition and study its relationship with amenability of $X$. Before doing so, we first consider a number of possible candidates for $X$, introduce a semi-norm on $M(S)$ and study its properties.

Let $B M(S)$ be the Banach space of all bounded (real) Borel measurable functions on $S$ with supermum norm. As usual, we define the left and right translations $l_{a} f, r_{a} f, f \in B M(S), a \in S$, by $l_{a} f(x)=f(a x), r_{a} f(x)$ $=f(x a), x \in S$. Let $C B(S)$ be the closed subspace of $B M(S)$ consisting of all bounded continuous functions,

$$
U C B_{r}(S)=L U C(S)=\left\{f \in C B(S): s \rightarrow l_{s} f \text { is norm continuous }\right\}
$$


and

$$
W L U C(S)=\left\{f \in C B(S): s \rightarrow l_{s} f \text { is weakly continuous }\right\} .
$$

Define $U C B_{l}(S)=R U C(S)$ and $W R U C(S)$ similarly and put

$$
U C B(S)=U C B_{l}(S) \cap U C B_{r}(S) \text {. }
$$

(See Greenleaf [6], Namioka [14], Mitchell [13] and Wong [18] for more details of these definitions and notations.) Finally, let $A(S)$ and $W(S)$ denote as usual the space of all strongly almost periodic and weakly almost periodic functions in $C B(S)$, respectively. For $f \in C B(S), \mu \in$ $M(S)$, we define the left and right convolutions $l_{\mu} f=\mu \odot f$ and $r_{\mu} f=f \odot$ $\mu$ by

$$
\mu \odot f(s)=\int f(t s) d \mu(t) \text { and } f \odot \mu(s)=\int f(s t) d \mu(t), \quad s \in S .
$$

(See Williamson [16] and Wong [18].) It is known that all these spaces are left and right translation invariant and, with the exception of $B M(S)$, they are all topological left and topological right translation invariant. (If $f \in B M(S), \mu \in M(S), \mu \odot f$ can be defined by the above formula as a generalised function which might not be in $B M(S)$. See Wong [20].)

Now define a map $T: B M(S) \rightarrow M(S)^{*}$ by

$$
T f(\mu)=\int f d \mu, \quad f \in B M(S), \mu \in M(S) .
$$

It is easy to show that $T$ is an order preserving linear isometry of $B M(S)$ into $M(S)^{*}$ such that $T(1)=1$. Moreover, $T$ commutes with left and right translations: $T\left(l_{a} f\right)=l_{a}(T f), T\left(r_{a} f\right)=r_{a}(T f), f \in B M(S), a \in S$. Also $T$ commutes with left and right convolutions: $T\left(l_{\mu} f\right)=l_{\mu}(T f), T\left(r_{\mu} f\right)=$ $r_{\mu}(T f), f \in B M(S), \mu \in M(S)$, provided that $l_{\mu} f$ and $r_{\mu} f \in B M(S)$ again. (See Wong [18, §5] and [20, §3].) It follows that the concepts of left (and also right) invariant means on any one of these spaces, whether as a space of functions (as usually defined in Day [3] or Hewitt and Ross [8, §17]), or as a space of functionals (in $M(S)^{*}$, as defined in $\S 1$ here), coincide (via the embedding $T$ ). The same is true for topological left (and topological right) invariant means except for $B M(S)$.

Returning to a general linear subspace $X \subset M(S)^{*}$, we introduce the following definition:

Definition 2.4. For $\mu \in M(S)$, let

$$
\|\mu\|_{X}=\sup \{|F(\mu)|: F \in X,\|F\| \leq 1\} .
$$


LEMMA 2.5.

(1) $\|\mu\|_{X}$ is a semi-norm on $M(S),\|\mu\|_{X} \leq\|\mu\|$ and $|F(\mu)| \leq\|F\| \cdot\|\mu\|_{X}$ if $F \in X$.

(2) If $X$ contains $T\left(C_{0}(S)\right)$ [e.g. when $\left.X=T(C B(S))\right]$, then $\|\mu\|_{X}=\|\mu\|$.

(3) If $X$ contains $\left\{\chi_{E}: E\right.$ Borel $\}$, where $\chi_{E}$ is the characteristic functional of $E$ defined by $\chi_{E}(\mu)=\mu(E), \mu \in M(S)[$ e.g. when $X=T(B M(S))$ or $\left.M(S)^{*}\right]$, then $\|\mu\|_{X}$ is a norm equivalent to $\|\mu\|$.

(4) If $X$ is topological right invariant, then $\|\mu \odot F\| \leq\|\mu\|_{X} \cdot\|F\|$, $F \in X, \mu \in M(S)$.

Proof.

(1) Straightforward.

(2) If $X$ contains $T\left(C_{0}(S)\right)$, then

$$
\begin{aligned}
\|\mu\|_{X} & =\sup \{|F(\mu)|: F \in X,\|F\| \leq 1\} \\
& \geq \sup \left\{\left|\int f d \mu\right|: f \in C_{0}(S),\|f\|_{u} \leq 1\right\}=\|\mu\|
\end{aligned}
$$

since $T$ is an isometry.

(3) If $X$ contains $\left\{\chi_{E}: E\right.$ Borel $\}$, then

$$
\|\mu\|_{X}=\sup \{|F(\mu)|: F \in X,\|F\| \leq 1\} \geq \sup \{|\mu(E)|: E \text { Borel }\},
$$

which is a norm on $M(S)$ equivalent to $\|\mu\|$ by [4, §III.7.4. p. 161].

(4) If $X$ is topological right invariant, then

$$
\begin{aligned}
\|\mu \odot F\| & =\sup \{|(\mu \odot F)(\nu)|:\|\nu\| \leq 1\}=\sup \{|(F \odot \nu)(\mu)|:\|\nu\| \leq 1\} \\
& \leq \sup \left\{\|F \odot \nu\| \cdot\|\mu\|_{X}:\|\nu\| \leq 1\right\} \leq\|F\| \cdot\|\mu\|_{X} \quad \text { if } F \in X .
\end{aligned}
$$

We now present

THEOREM 2.6. Let $X$ be a topological left invariant [left invariant] linear subspace of $M(S)^{*}$ containing the constant functional 1. Suppose, in addition, $X$ is either topological right invariant or $\|\mu\|_{X}$ is equivalent to $\|\mu\|$. Then $\Re_{0}(X)\left[\Re_{1}(X)\right]$ is closed under addition if the following condition is satisfied:

$\left(\mathrm{A}_{0}\right) \quad$ For any $\mu_{1}, \mu_{2} \in M_{0}(S)$,

$$
\begin{aligned}
& d_{X}\left(\mu_{1} * M_{0}(S), \mu_{2} * M_{0}(S)\right) \\
& \quad=\inf \left\{\left\|\mu_{1} * \mu-\mu_{2} * \nu\right\|_{X}: \mu, \nu \in M_{0}(S)\right\}=0 .
\end{aligned}
$$


$\left(\mathrm{A}_{1}\right) \quad\left[\right.$ For any $\mu_{1}, \mu_{2} \in M_{1}(S)$,

$$
\begin{aligned}
& d_{X}\left(\mu_{1} * M_{1}(S), \mu_{2} * M_{1}(S)\right) \\
& \left.\quad=\inf \left\{\left\|\mu_{1} * \mu-\mu_{2} * \nu\right\|_{X}: \mu, \nu \in M_{1}(S)\right\}=0 .\right]
\end{aligned}
$$

Proof. Suppose $\left(\mathrm{A}_{0}\right)$ is satisfied. Let $F_{1}, F_{2} \in \mathfrak{T}_{0}(X)$. For each $n$, there are $\mu_{1}, \mu_{2} \in M_{0}(S)$ such that $\left\|\mu_{i} \odot F_{i}\right\| \leq 1 / n$ for $i=1,2$. By $\left(\mathrm{A}_{0}\right)$, there are $\mu, \nu \in M_{0}(S)$ such that $\left\|\mu_{1} * \mu-\mu_{2} * \nu\right\|_{X} \leq 1 / n$. Put $\eta_{n}=$ $\mu_{1} * \mu \in M_{0}(S)$ and $G_{n}=\eta_{n} \odot\left(F_{1}+F_{2}\right)$. Then

$$
\begin{aligned}
G_{n} & =\eta_{n} \odot\left(F_{1}+F_{2}\right)=\eta_{n} \odot F_{1}+\eta_{n} \odot F_{2} \\
& =\mu \odot\left(\mu_{1} \odot F_{1}\right)+\left(\mu_{1} * \mu-\mu_{2} * \nu\right) \odot F_{2}+\nu \odot\left(\mu_{2} \odot F_{2}\right) .
\end{aligned}
$$

As a result

$$
\begin{aligned}
\left\|G_{n}\right\| & \leq\left\|\mu_{1} \odot F_{1}\right\|+\left\|\mu_{2} \odot F_{2}\right\|+\left\|\left(\mu_{1} * \mu-\mu_{2} * \nu\right) \odot F_{2}\right\| \\
& \leq 2 / n+C\left\|\mu_{1} * \mu-\mu_{2} * \nu\right\|_{X} \cdot\left\|F_{2}\right\|
\end{aligned}
$$

for some constant $C$ by Lemma 2.5. Therefore $\left\|G_{n}\right\| \leq 2 / n+(C / n)\left\|F_{2}\right\|$ $\rightarrow 0$ as $n \rightarrow \infty$. Thus $F_{1}+F_{2} \in \Re_{0}(X)$. Similarly for $\Re_{1}(X)$.

\section{REMARKS.}

(1) Theorem 2.6 is applicable to any of the spaces we considered above since they are all topological left and right invariant except $B M(S)$, which contains $T\left(C_{0}(S)\right)$.

(2) If the norms $\|\mu\|_{X}$ and $\|\mu\|$ are equivalent, then $\left(\mathrm{A}_{0}\right)$ is equivalent to the requirement that for each $\mu_{1}, \mu_{2} \in M_{0}(S), 0$ is in the weak closure of the convex set $\mu_{1} * M_{0}(S)-\mu_{2} * M_{0}(S)$.

(3) For the case of $\mathfrak{\varkappa}_{1}(X)$ in Theorem 2.6, we are unable to relax the condition of topological right invariant to right invariance only.

The converse of Theorem 2.3 for $\mathfrak{T}_{0}(X)$ is valid if $X$ is also topological left introverted: $M_{L}(X) \subset X$ for any mean $M$ on $X$ where $M_{L}(F)(\mu)=M(\mu \odot F), \mu \in M(S), F \in X$. For such $X$, an Arens product $M \odot N$ can be defined in $X^{*}$ by putting

$$
(M \odot N)(F)=M\left(N_{L}(F)\right), \quad F \in X, M, N \in X^{*} .
$$

Also topological left introvertedness implies topological right invariance. (Take $M=\nu \in M_{0}(S)$.) If $X$ has a topological left invariant mean and $F \in X$, we say $F$ is $X$-topological left almost convergent ( $X$-tlac) to the number $\beta$ if $M(F)=\beta$ for any topological left invariant mean $M$ on $X$. (See Wong $[17, \S 7]$ for the case of groups.) 
THEOREM 2.7. Let $X$ be a topological left introverted and topological left invariant linear sublattice of $M(S)^{*}$ containing the constant functionals. Then the following are equivalent:

(1) X has a topological left invariant mean.

(2) $\Re_{0}(X)$ is closed under addition.

In this case $\mathscr{T}_{0}(X)=\{F \in X: F$ is $X$-tlac to 0$\}=\mathcal{H}_{L}^{-}$, where $\mathcal{H}_{L}$ is the linear space spanned by $\mu \odot F-F, F \in X, \mu \in M_{0}(S)$ and the closure is taken in the norm (or weak) topology of $X$.

\section{Proof.}

(2) implies (1). This is a special case of Theorem 2.3.

(1) implies (2). It is clear that each $F \in \Re_{0}(X)$ is $X$-tlac to 0 . On the other hand, if $F \in X$ is $X$-tlac to 0 , we claim that $F \in \mathcal{H}_{L}^{-}$. Otherwise, by the Hahn-Banach Theorem, there is some $M \in X^{*}$ such that $M(F) \neq 0$ but $M\left(\mathcal{H}_{L}^{-}\right)=0$. Hence $M$ is topological left invariant. Now write $M=$ $C_{1} M_{1}-C_{2} M_{2}$, where $M_{1}, M_{2}$ are means on $X$, and let $N$ be a topological left invariant mean on $X$ (which exists by assumption). Then $M=N \odot M$ $=C_{1} N \odot M_{1}-C_{2} N \odot M_{2}$. But $N \odot M_{1}$ and $N \odot M_{2}$ are topological left invariant means on $X$ (since $N$ is). Hence $M(F)=C_{1}\left(N \odot M_{1}\right)(F)$ $C_{2}\left(N \odot M_{2}\right)(F)=0$ since $F$ is $X$-tlac to 0 . This is a contradiction. Thus $F \in \mathcal{H}_{L}^{-}$. Finally, $\mathcal{H}_{L}^{-} \subset \mathfrak{T}_{0}(X)$. To show this, consider functionals in $\mathcal{H}_{L}^{-}$ of the form $F_{1}=\mu \odot F-F$ with $F \in X, \mu \in M_{0}(S)$. Since $X$ has a topological left invariant mean, there is a net $\mu_{\alpha} \in M_{0}(S)$ such that $\mu * \mu_{\alpha}-\mu_{\alpha} \rightarrow 0$ in the weak topology $\sigma\left(X^{*}, X\right)$. Since $X$ is topological left introverted, for each $M \in X^{*}, M_{L}(F) \in X$. Hence

$$
M\left(\mu_{\alpha} \odot F_{1}\right)=M\left(\left(\mu * \mu_{\alpha}-\mu_{\alpha}\right) \odot F\right)=M_{L}(F)\left(\mu * \mu_{\alpha}-\mu_{\alpha}\right) \rightarrow 0
$$

for any $M \in X^{*}$. That is, $0 \in \sigma\left(X, X^{*}\right)$, hence norm, closure in $X$ of $M_{0}(S) \odot F_{1}$. Therefore $F_{1} \in \Re_{0}(X)$. Since the net $\mu_{\alpha}$ is independent of $F_{1}$, it follows that $\mathcal{H}_{L} \subset \mathscr{T}_{0}(X)$, which is obviously closed.

Thus $\mathcal{H}_{L}^{-}=\mathscr{T}_{0}(X)=\{F \in X: F$ is $X$-tlac to 0$\}$. Consequently, $\Re_{0}(X)$ is closed under addition.

\section{REMARKS.}

(1) If $X=M(S)^{*}$, Theorem 2.7 is an analogue of a result in Emerson [5, Theorem 1.7, (a) iff (b)] for groups. In fact, we have obtained more here by identifying $\mathcal{T}_{0}(X), \mathcal{H}_{L}^{-}$and the $X$-topological left almost convergent to 0 functionals, thereby unifying the results. 
(2) For $\Re_{1}(X)$, Theorem 2.7 is not generally available simply because the "left introversion" $M_{l}(F)$ can only be defined as a function $M_{l}(F)(s)$ $=M\left(l_{s} F\right)$ and not a functional in $M(S)^{*}$. When $X$ contains only functions, Theorem 2.7 can be formulated for $\Re_{1}(X)$ using left introversion, but then this is also covered under Theorem 2.7 by considering $X \subset$ $M\left(S_{d}\right)$ * where $S_{d}$ is $S$ with the discrete topology.

(3) For locally compact groups $G$ and functions in $L_{\infty}(G)$, we have a collection of similar results. See $\$ 4$ below.

The converse of Theorem 2.6 is not true even if $X$ is topological left introverted. Consider $X=T(W(S))$. If $m \in W(S)^{*}$ and $f \in W(S)$, we define the left introversion $m_{l}(f)$ by $m_{l}(f)(s)=m\left(l_{s} f\right), s \in S$. It is well known that $W(S)$ is left introverted: $m_{l}(W(S)) \subset W(S)$, for any $m \in$ $W(S)^{*}$. Moreover $m(\mu \odot f)=\int m_{l}(f) d \mu$. (In fact, the same holds for $f \in W L U C(S)$. See Kharaghani [11].) Therefore if $M \in X^{*}, f \in W(S)$, $\mu \in M(S)$, we have

$$
\begin{aligned}
M_{L}(T f)(\mu) & =M(\mu \odot T f)=M T(\mu \odot f)=m(\mu \odot f) \\
& =\int m_{l}(f) d \mu=T\left(m_{l}(f)\right)(\mu) \quad \text { where } m=T^{*} M
\end{aligned}
$$

Thus $M_{L}(T f)=T\left(m_{l}(f)\right)$ and $X$ is topological left introverted in $M(S)^{*}$. If $S=G$ is a locally compact group, then $X$ or rather $W(G)$ always has a unique topological left invariant mean whether $G$ is amenable or not. Hence by Theorem 2.7, $\Re_{0}(X)$ is closed under addition. If $\left(\mathrm{A}_{0}\right)$ is satisfied for $X=T(W(G)) \supset T\left(C_{0}(G)\right)$, then $\left(\mathrm{A}_{0}\right)$ is also satisfied for $X=M(G)^{*}$ since $\|\mu\|=\|\mu\|_{X}$. By Theorem 2.6, $M(G)^{*}$ must have a topological left invariant mean, which is not true in general.

However, for topological right full subspaces $X$ of $M(S)^{*}$, the converses of both Theorems 2.3 and 2.6 are true (Theorem 2.12).

Definition 2.8. A subset $X \subset M(S)^{*}$ is called topological right full in $M(S)^{* *}$ if for each $\mu_{1}, \mu_{2} \in M_{0}(S)$, the following is true: there is a net $\mu_{\alpha}$ (depending on $\left.\mu_{1}, \mu_{2}\right)$ in $M_{0}(S)$ such that $\left(\mu_{1}-\mu_{2}\right) * \mu_{\alpha} \rightarrow 0$ in the topology $\sigma(M(S), X)$ iff $0 \in$ weak (or norm) closure of the convex set $\left(\mu_{1}-\mu_{2}\right) * M_{0}(S)$ in $M(S)$. (See Emerson [5] for the concept of full subspaces in $L_{\infty}(G)$.)

The next two lemmas have their counterparts in Emerson [5] for groups. The proof, which we shall omit, are straightforward modifications of those in [5, Proposition 2.1 and Corollary 2.2]. 
LEMMA 2.9. If $Y$ is a linear subpsace of $M(S)^{*}$, then there is a mean $M$ on $M(S)^{*}$ such that $M(Y)=0$ iff $\inf \left\{F(\mu): \mu \in M_{0}(S)\right\} \leq 0$ for all $F \in Y$.

LEMMA 2.10. For each $F \in M(S)^{*}$, there is a mean $M_{F}$ on $M(S)^{*}$ such that

$$
M(\mu \odot F)=M_{F}(F) \quad \text { for all } \mu \in M_{0}(S)
$$

iff

$$
\inf \left\{\left(\mu_{1}-\mu_{2}\right) \odot F(\nu): \nu \in M_{0}(S)\right\} \leq 0 \text { for any } \mu_{1}, \mu_{2} \in M_{0}(S) .
$$

Proposition 2.11. If $X$ is a topological right full linear subspace of $M(S)^{*}$, then the following are equivalent:

(a) $M(S)^{*}$ has a topological left invariant mean.

(b) There is a mean $M$ on $M(S)^{*}$ such that $M(\mu \odot F)=M(F)$ for all $\mu \in M_{0}(S)$ and $F \in X$.

(c) For each $F \in X$, there is a mean $M_{F}$ on $M(S)^{*}$ such that $M_{F}(\mu \odot F)=M_{F}(F)$ for all $\mu \in M_{0}(S)$.

Proof. We need only show (c) implies (a). Let $\mu_{1}, \mu_{2} \in M_{0}(S)$ and consider $Y=\left(\mu_{1}-\mu_{2}\right) \odot X$. By (c) and Lemma 2.10, for any $F \in X$, $\inf \left\{\left(\mu_{1}-\mu_{2}\right) \odot F(\nu): \nu \in M_{0}(S)\right\} \leq 0$. By Lemma 2.9, there is some mean $M$ on $M(S)^{*}$ such that $M(Y)=0$. Let $\mu_{\alpha}$ be a net in $M_{0}(S)$ such that $\mu_{\alpha} \rightarrow M$ in weak* topology of $M(S)^{* *}$. Then for any $F \in X$,

$$
F\left(\left(\mu_{1}-\mu_{2}\right) * \mu_{\alpha}\right)=\left(\mu_{1}-\mu_{2}\right) \odot F\left(\mu_{\alpha}\right) \rightarrow M\left(\left(\mu_{1}-\mu_{2}\right) \odot F\right)=0 .
$$

Thus $0 \in \sigma(M(S), X)$ closure of $\left(\mu_{1}-\mu_{2}\right) * M_{0}(S)$. Since $X$ is topological right full, $0 \in$ norm closure of $\left(\mu_{1}-\mu_{2}\right) * M_{0}(S)$ and a fortiori, of $\mu_{1} * M_{0}(S)-\mu_{2} * M_{0}(S)$. That is, $d\left(\mu_{1} * M_{0}(S), \mu_{2} * M_{0}(S)\right)=0$. By Theorem 2.6, applied to $M(S)^{*}$, this implies (a).

THEOREM 2.12. Let $X$ be a topological left invariant linear subspace of $M(S)^{*}$ containing 1. Consider the following conditions:

(a) $X$ has a topological left invariant mean.

(b) $\Re_{0}(X)$ is closed under addition.

(c) $d_{X}\left(\mu_{1} * M_{0}(S), \mu_{2} * M_{0}(S)\right)=0$ for any $\mu_{1}, \mu_{2}$ in $M_{0}(S)$.

If $X$ is topological right full, then (a) and (b) are equivalent (thus converse of Theorem 2.3 holds) and each implies (c).

If $X$ is topological right full and either \|\|$\sim\|\|_{X}$ or $X$ is topological right invariant, then all three are equivalent. (Hence the converse of Theorem 2.6 holds.) 
Proof. By Theorem 2.3, (b) always implies (a). Let $X$ be topological right full and assume (a). By Proposition $2.11, M(S)^{*}$ has a topological left invariant mean. Hence $\Re_{0}(S)=\Re_{0}\left(M(S)^{*}\right)$ is closed under addition by Theorem 2.7 (applied to $\left.M(S)^{*}\right)$. So is $\mathfrak{N}_{0}(X)=\mathfrak{N}_{0}(S) \cap X$ and (a) implies (b). On the other hand, (a) also implies there is a net $\mu_{\alpha} \in M_{0}(S)$ such that $\left\|\mu * \mu_{\alpha}-\mu_{\alpha}\right\| \rightarrow 0$ for all $\mu \in M_{0}(S)$ (since $M(S)^{*}$ has a topological left invariant mean). Therefore

$$
d_{X}\left(\mu_{1} * M_{0}(S), \mu_{2} * M_{0}(S)\right) \leq d\left(\mu_{1} * M_{0}(S), \mu_{2} * M_{0}(S)\right)=0
$$

for any $\mu_{1}, \mu_{2} \in M_{0}(S)$ or (a) implies (c). The last assertion of the theorem follows from Theorem 2.6.

REMARKs. (1) If $X=M(S)^{*}$, then (a), (b) and (c) are equivalent. This is a semigroup analogue of a result in Emerson [5, Theorem 1.7] for groups. Moreover, if any one of these holds, then $\Re_{0}(S)=\Re_{0}\left(M(S)^{*}\right)$ consists of all $F \in M(S)^{*}$ which are topological left almost convergent to zero in $M(S)^{*}$, which in turn coincides with the closure of the linear span $\mathcal{H}_{L}$ of $\left\{\mu \odot F-F, \mu \in M_{0}(S), F \in M(S)^{*}\right\}$ in $M(S)^{*}$ (Theorem 2.7).

(2) There are versions of Lemmas 2.9 and 2.10 for left invariant means, and right full linear subpsace of $M(S)^{*}$ can be defined. However, we are unable to obtain the corresponding versions of Proposition 2.11 and Theorem 2.12. The difficulty seems to lie in the fact that, in general, $M_{1}(S)$ might not be $\sigma\left(X^{*}, X\right)$ dense in the set of means on $X$ if $X$ contains functionals not coming from functions.

(3) Proposition 2.11 implies that if $X$ is a topological right full linear subspace of $M(S)^{*}$, then $X$ has a topological left invariant mean iff $M(S)^{*}$ does. Moreover, if we put $X=M(S)^{*}$, then Proposition 2.11 becomes the well-known Localisation Theorem (see also Emerson [5, Theorem 2.3, p. 188]).

3. Some examples. It remains to find examples of topological left introverted and topological right full subspaces of $M(S)^{*}$. As mentioned earlier, $W(S)$ and $M(S)^{*}$ are always topological left introverted. So are $W L U C(S)$ and $L U C(S)$, but $C B(S)$ in general is not. (See Kharaghani [11, Chapter II, §2.2, p. 13] for more details.)

On the other hand $W(S)$ in general is not topological right full. For example, take $S$ to be any locally compact group which is not amenable. In what follows, we shall give a sufficient condition for a subspace $X$ of $M(S)^{*}$ to be topological right full and in particular show that $C B(S)$ is topological right full for "all" locally compact subsemigroups of any locally compact group. 
THEOREM 3.1. $X$ is a topological right full subspace of $M(S)^{*}$ if for each $F \in M(S)^{*}$ there is some $\mu \in M_{0}(S)$ (possibly depending on $F$ ) such that $F \odot \mu \in X$.

Proof. Suppose $\mu_{1}, \mu_{2} \in M_{0}(S)$ and $\mu_{\alpha}$ is a net in $M_{0}(S)$ such that $\left(\mu_{1}-\mu_{2}\right) * \mu_{\alpha} \rightarrow 0$ in the topology $\sigma(M(S), X)$. Let $F \in M(S)^{*}$. By assumption, there is some $\mu \in M_{0}(S)$ such that $F \odot \mu \in X$. For each $n$, there is some $\mu_{\alpha_{n}}$ such that

$$
\left.\mid(F \odot \mu)\left(\mu_{1}-\mu_{2}\right) * \mu_{\alpha_{n}}\right) \mid<1 / n \text { or }\left|F\left(\left(\mu_{1}-\mu_{2}\right) * \nu_{n}\right)\right|<1 / n
$$

where $\nu_{n}=\mu_{\alpha_{n}} * \mu \in M_{0}(S)$. Therefore for each $F \in M(S)^{*}$, there is a sequence in $M_{0}(S)$ (possibly depending on $F, \mu_{1}, \mu_{2}$ ) such that $F\left(\left(\mu_{1}-\mu_{2}\right) * \nu_{n}\right) \rightarrow 0$. By Emerson [5, Proposition 2.5 applied to the convex set $\left.C=\left(\mu_{1}-\mu_{2}\right) * M_{0}(S)\right]$, this implies there is a net $\nu_{\beta} \in M_{0}(S)$ (depending on $\mu_{1}, \mu_{2}$ but not $F$ ) such that $\left(\mu_{1}-\mu_{2}\right) * \nu_{\beta} \rightarrow 0$ in weak topology of $M(S)$. Consequently, $X$ is topological right full.

REMARK. In [5] Emerson also gives a sufficient condition for right fullness of subspaces in $L_{\infty}(G)$ which involves an approximate identity of the locally compact group $G$.

Definition 3.2. A measure $\mu$ in $M(S)$ is said to be left absolutely continuous if the map $s \rightarrow \varepsilon_{s} * \mu$ is norm continuous. The set of all left absolutely continuous measures in $M(S)$ is denoted by $M_{a}^{l}(S)$. (See Hart [7] and Wong [19]. For groups $M_{a}^{l}(G)=M_{a}(G)=L_{1}(G)$, see Hewitt and Ross [8].)

THEOREM 3.3. If $\left.M_{a}^{\mathrm{l}}(S) \cap M_{(} S\right) \neq \varnothing$, then $T(R U C(S))$ is a topological right full subspace of $M(S)^{*}$.

Proof. Let $\mu \in M_{a}^{l}(S) \cap M_{0}(S)$ be fixed. For any $F \in M(S)^{*}$, we set $f(s)=F\left(\varepsilon_{s} * \mu\right)$. Then

$$
\left\|r_{s} f-r_{t} f\right\|_{u} \leq\|F\| \cdot\left\|\varepsilon_{s} * \mu-\varepsilon_{t} * \mu\right\|
$$

and hence $f \in R U C(S)$. By Wong [20, Lemma 4.5, p. 92], $F(\nu * \mu)=$ $\int F\left(\varepsilon_{s} * \mu\right) d \nu$ for any $\nu \in M(S)$. That is, $F \odot \mu=T f \in T(R U C(S))$. By the preceding theorem, $T(R U C(S))$ is topological right full.

Note. In Wong [20] the semigroups are assumed to be jointly continuous. But the results there are also valid for the separately continuous case. See Wong [21]. 
If we define $M_{w a}^{l}(S)$, the weakly left absolutely continuous measures, as $\left\{\mu \in M(S): s \rightarrow \varepsilon_{s} * \mu\right.$ is weakly continuous $\}$, then we have

THeOREM 3.4. If $M_{w a}^{l}(S) \cap M_{0}(S) \neq \varnothing$, then $T(C B(S))$ is a topological right full subspace of $M(S)^{*}$.

Proof. Similar to that of Theorem 3.3 with straightforward modifications.

THEOREM 3.5. Let $S$ be a locally compact Borel subsemigroup of a locally compact group $G$. If $S$ contains a non-empty open subset of $G$, then $M_{a}^{l}(S) \cap M_{0}(S) \neq \varnothing$.

Proof. Let $A \neq \varnothing$ be an open set in $G$ such that $A \subset S$. Choose any open neighbourhood $U$ of the identity $e$ in $G$ with $0<\lambda(U)<\infty$, where $\lambda$ is the left Haar measure, and fix $y \in A$. Consider $B=A \cap y U \neq \varnothing . B$ is open and $B \subset S$. Moreover, $0<\lambda(B) \leq \lambda(y U)=\lambda(U)<\infty$. By Wong $\left[19, \S 4\right.$, p. 131], $M_{a}^{l}(S) \cap M_{0}(S) \neq \varnothing$.

4. Locally compact groups. Let $G$ be a locally compact group. Consider the space $L_{\infty}(G)=L_{1}(G)^{*}$ with $L_{1}(G) \cong M_{a}(G) \subset M(G)$, where $M_{a}(G)$ is the ideal in $M(G)$ of all absolutely continuous measures (with respect to a fixed left Haar measure $\lambda$ ). If $f \in L_{\infty}(G), \mu \in M(G)$, we can define the convolutions $l_{\mu} f=\mu \odot f$ and $r_{\mu} f=f \odot \mu$ by

$$
\mu \odot f=\tilde{\mu} * f \text { and } f \odot \mu=f * \nu
$$

where $\tilde{\mu}(E)=\mu\left(E^{-1}\right), E$ Borel, $d \nu=\Delta d \tilde{\mu}$ and $\Delta$ is the modular function. (See Hewitt and Ross [8, Theorems 20.12 and 20.13].) Thus $\mu \odot f$ and $f \odot \mu$ are defined in such a way that they coincide with those in $\$ 2$ in the case when $f \in C B(G)$. If $\mu \in M_{a}(G) \cong L_{1}(G)$, then $\mu \odot f=\Delta^{-1} \tilde{\varphi} * f$ and $f \odot \mu=f * \tilde{\varphi}$, where $\varphi \in L_{1}(G)$ is such that $d \mu=\varphi d \lambda$ and $\tilde{\varphi(x)}=$ $\varphi\left(x^{-1}\right)$. (See Emerson [5] and Hewitt and Ross [8] where the notation $\varphi^{*}$ is used in place of $\varphi^{\tilde{y}}$.) If $X$ is any linear subspace of $L_{\infty}(G)$ containing 1 , a mean $m$ on $X$ is defined as usual as an element $m \in X^{*}$ such that for each $f \in X$, essinf $f \leq m(f) \leq \operatorname{ess} \sup f$, or, equivalently,

$$
\inf \{(f, \varphi): \varphi \in P(G)\} \leq m(f) \leq \sup \{(f, \varphi): \varphi \in P(G)\},
$$

where $(f, \varphi)$ is the natural pairing of $L_{\infty}(G)$ and $L_{1}(G)$ and $P=P(G)=$ $\left\{\varphi \in L_{1}(G): \varphi \geq 0,\|\varphi\|_{1}=1\right\}$. There are now two definitions of topological left invariance. Let $X$ be a topological left invariant (i.e. $l_{\mu}(X)$ for all $\mu \in M_{0}(G)$ ) linear subspace of $L_{\infty}(G)$ containing the constants. A 
mean $m$ on $X$ is called topological left invariant if $m(\mu \odot f)=m(f)$ for any $f \in X$ and $\mu \in M_{0}(G)$. Similarly, we can define the topological left $P(G)$-invariant mean on topological left $P(G)$-invariant subspaces $X$ of $L_{\infty}(X)$. However, topological left $P(G)$-invariance of $X$ may not imply topological left invariance of $X$. (The converse implication is of course true.) It is well known that if $X$ is topological left invariant, then two definitions of topological left invariant and topological left $P(G)$-invariant means coincide because $L_{1}(G)$ is an ideal in $M(G)$. (See A. Hulanicki [9].) Thus, we lose very little by considering only topological left $P(G)$-invariant means. (See Wong [17] where they are simply called topological left invariant.) Now define

$$
\begin{aligned}
& \Re_{P}(X)=\{f \in X: 0 \in \text { norm or weak closure } \\
& \qquad \text { of the convex set } P(G) * f\}, \\
& \|\varphi\|_{X}=\sup \left\{|(f, \varphi)|: f \in X,\|f\|_{\infty} \leq 1\right\}
\end{aligned}
$$

and

$$
d_{X}\left(\varphi_{1} * P, \varphi_{2} * P\right)=\inf \left\{\left\|\varphi_{1} * \varphi-\varphi_{2} * \psi\right\|_{X}: \varphi, \psi \in P\right\} .
$$

Also, we can define topological left introvertion $\left(m_{L}(f)(\varphi)=m\left(l_{\varphi} f\right)\right.$, $m \in X^{*}, f \in X, \varphi \in L_{1}(G)$ ), and if $X$ is topological left introverted in $L_{\infty}(G)\left(m_{L}(X) \subset X\right.$ for any $\left.m \in X^{*}\right)$, an Arens product on $X^{*}$ can be defined by $(m \odot n)(f)=m\left(n_{L}(f)\right), m, n \in X^{*}, f \in X$. Finally, we can define topological right fullness in the obvious way. Then all the results in $\$ 2$ can be carried over to the present context. In particular, if $X=L_{\infty}(G)$, we recover most of the major results in Emerson [5], plus the fact that $\Re_{p}\left(L_{\infty}(G)\right)$ consists of all functions in $L_{\infty}(G)$ which are topological left almost convergent to 0 , which in turn coincide with the closure of the linear span of $\left\{\varphi * f-f, \varphi \in P(G), f \in L_{\infty}(G)\right\}$ provided that $G$ is amenable. We omit the details. (See also Wong [17].)

What we would like to do here, as mentioned in the abstract, is to provide an answer to an open problem raised in Emerson [5, p. 187]. As suggested there, we can also consider $\mathscr{T}_{1}\left(L_{\infty}(G)\right)=\left\{f \in L_{\infty}(G): 0 \in\right.$ weak or norm closure of $\left.M_{1}(G) \odot f\right\}$ and show that if $d\left(\mu_{1} * M_{1}(G)\right.$, $\left.\mu_{2} * M_{1}(G)\right)=0$ for any $\mu_{1}, \mu_{2} \in M_{1}(G)$, then $L_{\infty}(G)$ must have a left invariant mean. The converse is, however, false since the former condition implies that $G$ is amenable as a discrete group. The problem is to find a suitable replacement equivalent to amenability of $G$ as a locally compact group. The answer is provided in the next theorem (Condition (d)). 
THEOREM 4.1. Let $G$ be a locally compact group. Then the following are equivalent:

(a) $L_{\infty}(G)$ has a (topological) left invariant mean.

(b) $U C B(G)$ has a (topological) left invariant mean.

(c) $\mathfrak{T}_{1}(U C B(G))$ is closed under addition.

(d) For any $\mu_{1}, \mu_{2} \in M_{1}(G), 0 \in \sigma(M(G), U C B(G))$ closure of the convex set $\mu_{1} * M_{1}(G)-\mu_{2} * M_{1}(G)$.

Proof. It is well known that (a) and (b) are equivalent. (b) and (c) are equivalent by Theorem 2.7 applied to $X=U C B(G)$ or rather, $T(U C B(G))$, considered as a topological left introverted and topological left invariant linear subspace of $M\left(G_{d}\right)^{*}=m(G)$, the bounded functions on $G$, where $G_{d}$ is the group $G$ with the discrete topology. To show that (b) implies (d), suppose $U C B(G)$ has a left invariant mean. Then there is a net $\mu_{\alpha} \in M_{1}(G)$ such that $\mu * \mu_{\alpha}-\mu_{\alpha} \rightarrow 0$ in $\sigma(M(G), U C B(G))$ for any $\mu \in M_{1}(G)$. This certainly implies (d). Conversely, assume (d). We want to prove (c). Take $f_{1}, f_{2} \in \mathcal{N}_{1}(U C B(G))$. Then there are nets $\mu_{1}^{\alpha}, \mu_{2}^{\alpha} \in M_{1}(G), \alpha \in D$, such that $\mu_{1}^{\alpha} \odot f_{1} \rightarrow 0, \mu_{2}^{\alpha} \odot f_{2} \rightarrow 0$ in the norm topology of $U C B(G)$. (Notice we can assume $\mu_{1}^{\alpha}, \mu_{2}^{\alpha}$ have the same directed set $D$; otherwise take the product of their directed sets.) By assumption, for each $\alpha \in D$, there is a directed set $E_{\alpha}$ and nets $\mu_{\beta}^{\alpha}, \nu_{\beta}^{\alpha} \in M_{1}(G), \beta \in E_{\alpha}$, such that $\mu_{1}^{\alpha} * \mu_{\beta}^{\alpha}-$ $\mu_{2}^{\alpha} * \nu_{\beta}^{\alpha} \rightarrow 0$ in $\sigma(M(G), U C B(G))$ with respect to $\beta$. For each $(\alpha, g)$ in the product directed set $D \times \Pi\left\{E_{\alpha}: \alpha \in D\right\}$, we define $R(\alpha, g)=(\alpha, g(\alpha))$, $\alpha \in D, g \in \Pi\left\{E_{\alpha}: \alpha \in D\right\}$ and let

$$
S(\alpha, \beta)=\left(\mu_{1}^{\alpha} * \mu_{\beta}^{\alpha}-\mu_{2}^{\alpha} * \nu_{\beta}^{\alpha}\right) \odot f_{2} \in U C B(G) .
$$

We claim that $\lim _{\beta} S(\alpha, \beta)=0$ in the weak topology of $U C B(G)$ for each $\alpha \in D$. Note that if $m \in U C B(G)^{*}, f \in U C B(G)$ and $\mu \in M_{d}(G)$, the discrete measures, then

$$
m(\mu \odot f)=\int m_{l}(f) d \mu
$$

where $m_{l}(f)(s)=m\left(l_{s} f\right), s \in G$. (See also Kharaghani [11, Chapter II, Lemma 2.2.2, p. 14].) Using this and the fact that $U C B(G)$ is left introverted, we have, for each $m \in U C B(G)^{*}$,

$$
m(S(\alpha, \beta))=\int m_{l}\left(f_{2}\right) d\left(\mu_{1}^{\alpha} * \mu_{\beta}^{\alpha}-\mu_{2}^{\alpha} * \nu_{\beta}^{\alpha}\right),
$$

which converges to 0 with respect to $\beta$, for fixed $\alpha$. Therefore

$$
\lim _{\alpha} \lim _{\beta} S(\alpha, g)=0
$$


in the weak topology of $U C B(G)$. By the Iterated Limit Theorem (Kelley [10, Theorem 4, p. 69]),

$$
\lim _{(\alpha, g)} S \circ R(\alpha, g)=0 \quad \text { or } \quad \lim _{(\alpha, g)}\left(\mu_{1}^{\alpha} * \mu_{g(\alpha)}^{\alpha}-\mu_{2}^{\alpha} * \nu_{g(\alpha)}^{\alpha}\right) \odot f_{2}=0
$$

in the weak topology of $U C B(G)$.

On the other hand, for any $g$,

$$
\left\|\mu_{g(\alpha)}^{\alpha} \odot\left(\mu_{1}^{\alpha} \odot f_{1}\right)+\nu_{g(\alpha)}^{\alpha} \odot\left(\mu_{2}^{\alpha} \odot f_{2}\right)\right\| \leq\left\|\mu_{1}^{\alpha} \odot f_{1}\right\|+\left\|\mu_{2}^{\alpha} \odot f_{2}\right\| \stackrel{\alpha}{\rightarrow} 0 .
$$

Hence,

$$
\lim _{(\alpha, g)}\left\{\mu_{g(\alpha)}^{\alpha} \odot\left(\mu_{1}^{\alpha} \odot f_{1}\right)+\nu_{g(\alpha)}^{\alpha} \odot\left(\mu_{2}^{\alpha} \odot f_{2}\right)\right\}=0
$$

in the norm, hence weak, topology of $U C B(G)$. This means there is a net $\mu_{(\alpha, g)}=\mu_{1}^{\alpha} * \mu_{g(\alpha)}^{\alpha}$ in $M_{1}(G)$ such that

$$
\begin{aligned}
\mu_{(\alpha, g)} \odot\left(f_{1}+f_{2}\right)= & \mu_{g(\alpha)}^{\alpha} \odot\left(\mu_{1}^{\alpha} \odot f_{1}\right)+\mu_{g(\alpha)}^{\alpha} \odot\left(\mu_{1}^{\alpha} \odot f_{2}\right) \\
= & \mu_{g(\alpha)}^{\alpha} \odot\left(\mu_{1}^{\alpha} \odot f_{1}\right)+\nu_{g(\alpha)}^{\alpha} \odot\left(\mu_{2}^{\alpha} \odot f_{2}\right) \\
& +\left(\mu_{1}^{\alpha} * \mu_{g(\alpha)}^{\alpha}-\mu_{2}^{\alpha} * \nu_{g(\alpha)}^{\alpha}\right) \odot f_{2}
\end{aligned}
$$

converges to 0 with respect to $(\alpha, g)$ in the weak topology of $U C B(G)$. That is, $0 \in$ weak (hence norm) closure of the convex set $M_{1}(G) \odot\left(f_{1}+\right.$ $\left.f_{2}\right)$ or $f_{1}+f_{2} \in \Re_{1}(U C B(G))$. Thus $\Re_{1}(U C B(G))$ is closed under addition and (d) implies (c). This completes the proof.

\section{REMARKS.}

(1) The space $U C B(G)$ is chosen for convenience. All we need here is some $X \subset C B(G)$ which is left introverted and topological right full (in $\left.M(G)^{*}\right)$.

(2) In [5] Emerson considered the discrete measures $D=M_{d}(G) \cap$ $M_{0}(G)$ of weights one and defined $\Re_{D}$ accordingly. But $M_{1}(G)$ is norm dense in $D$. Hence $\Re_{D}=\Re_{1}$.

(3) If $\Re_{1}\left(L_{\infty}(G)\right)$ is closed under addition, then so is $\Re_{1}(U C B(G))$. The converse remains open $\left(L_{\infty}(G)\right.$ is not left introverted!).

Finally, in conclusion, we would like to point out that the space $L_{\infty}(G)$ can also be embedded into $M(G)^{*}$ by a lifting (the map $T$ does not work since elements of $L_{\infty}(G)$ are equivalence classes). Recall that a lifting $\rho$ is a map $\rho: B M(G) \rightarrow B M(G)$ satisfying:

(1) $\rho(f) \sim f$

(2) $f \sim g$ implies $\rho(f)=\rho(g)$; 
(3) $f \geq 0$ implies $\rho(f) \geq 0$;

(4) $\rho(1)=1$;

(5) $\rho$ is linear;

(6) $\rho$ is multiplicative (pointwise).

Here $f \sim g$ means $f-g$ is a $\lambda$-locally null function.

It is known that for any locally compact group $G$, there exists a lifting which commutes with left translations $\rho\left(l_{a} f\right)=l_{a}(\rho(f)), a \in G$. Such a lifting is necessarily strong (i.e. $\rho(f)=f$ for any $f \in C B(G)$ ). See Tulcea and Tulcea [15]. It follows that $\rho$ commutes with left convolutions $l_{\varphi}$, $\varphi \in L_{1}(G)$, since $\varphi \odot f \in C B(G)$, if $f \in B M(G)$. Define $\bar{T}: L_{\infty}(G) \rightarrow$ $M(G)^{*}$ by

$$
\bar{T}(\bar{f})=T(\rho(f)),
$$

where $\bar{f} \in L_{\infty}(G)$ is the equivalence class determined by $f \in B M(G)$. It is simple to show that $\bar{T}$ is an order preserving linear isometry of $L_{\infty}(G)$ into $M(G)^{*}$ which commutes with left translations and left convolutions $l_{\varphi}$, $\varphi \in L_{1}(G)$, and which "agrees" with $T$ on $B M(G)$ such that $\bar{T}(1)=1$. However, we do not know if $\bar{T}$ also commutes with left convolutions $l_{\mu}$ with $\mu \in M(G)$. Nevertheless, it follows that the concepts of left invariant means (respectively topological left $P(G)$-invariant means) on $L_{\infty}(G)$ and suitable subspaces of $L_{\infty}(G)$ agree whether as functions or functionals.

\section{REFERENCES}

[1] R. F. Arens, The adjoint of a bilinear operator, Proc. Amer. Math. Soc., 2 (1951), 839-848.

[2] S. Banach, Sur le problème de la mesure, Fund. Math., 4 (1923), 7-31.

[3] M. M. Day, Amenable semigroups, Illinois J. Math., 1 (1957), 509-544.

[4] N. Dunford and J. T. Schwartz, Linear Operators, Part I, Pure and Applied Math. No. 7, Interscience, New York, 1958.

[5] W. R. Emerson, Characterisations of amenable groups, Trans. Amer. Math. Soc., 241 (1978), 183-194.

[6] F. P. Greenleaf, Invariant Means On Topological Groups, Van Nostrand, Princeton, N.J., 1969.

[7] G. Hart, Absolute continuous measures on semigroups, Ph.D. thesis, Kansas State Univ., Kansas (1970).

[8] E. Hewitt and K. A. Ross, Abstract Harmonic Analysis I, Springer-Verlag, Berlin, 1963.

[9] A. Hulanicki, Means and Fǒlner conditions on locally compact groups, Studia Math., 27 (1966), 87-104.

[10] J. Kelley, General Topology, Van Nostrand, New York, 1955.

[11] H. Kharaghani, Invariant means on topological semigroups, Ph.D. thesis, Univ. of Calgary, Calgary, 1974. 
[12] T. Mitchell, Function algebras, means and fixed points, Trans. Amer. Math. Soc., 130 (1968), 117-126.

[13] _ Topological semigroups and fixed points, Illinois J. Math., 14 (1970), 630-641.

[14] I. Namioka, On certain actions of semigroups of L-spaces, Studia Math., 29 (1967), 63-77.

[15] I. Tulcea and C. Tulcea, On the existence of a lifting commuting with the left translations of an arbitrary locally compact group, Proceedings of the Fifth Berkeley Symposium on Mathematical Statistics and Probability, Vol. II, Part 1 (1966-67), 63-97.

[16] J. Williamson, Harmonic analysis on semigroups, J. London Math. Soc., 42 (1967), $1-14$.

[17] James C. S. Wong, Topological stationary locally compact groups and amenability, Trans. Amer. Math. Soc., 144 (1969), 351-363.

[18] An ergodic property of locally compact amenable semigroups, Pacific J. Math., 48 (1973), 615-619.

[19] Absolutely continuous measures on locally compact semigroups, Canad. Math. Bull., 18 (1975), 127-132.

[20] Abstract harmonic analysis on locally compact semigroups with application to invariant means, J. Australian math. Soc., 13 (Series A) Part 1 (1977), 84-94.

[21] _ Convolution and separate continuity, Pacific J. Math., 75 (1978), 601-611.

Received June 4, 1980. The first author was supported by the National Sciences and Engineering Research Council of Canada Grant No. A8227.

The University of CALGary

Calgary, Canada T2N 1N4 


\title{
PACIFIC JOURNAL OF MATHEMATICS EDITORS
}

\author{
Donald BabBitT (Managing Editor) \\ University of California \\ Los Angeles, CA 90024 \\ Hugo Rossi \\ University of Utah \\ Salt Lake City, UT 84112 \\ C. C. Moore and Arthur Ogus \\ University of California \\ Berkeley, CA 94720
}

\author{
J. DugundiI \\ Department of Mathematics \\ University of Southern California \\ Los Angeles, CA 90089-1113 \\ R. FINN and H. SAMELSON \\ Stanford University \\ Stanford, CA 94305
}

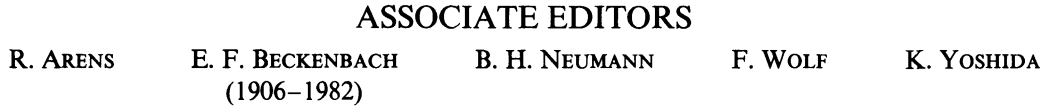

\section{SUPPORTING INSTITUTIONS}

UNIVERSITY OF ARIZONA
UNIVERSITY OF BRITISH COLUMBIA
CALIFORNIA INSTITUTE OF TECHNOLOGY
UNIVERSITY OF CALIFORNIA
MONTANA STATE UNIVERSITY
UNIVERSITY OF NEVADA, RENO
NEW MEXICO STATE UNIVERSITY
OREGON STATE UNIVERSITY

\author{
UNIVERSITY OF OREGON \\ UNIVERSITY OF SOUTHERN CALIFORNIA \\ STANFORD UNIVERSITY \\ UNIVERSITY OF HAWAII \\ UNIVERSITY OF TOKYO \\ UNIVERSITY OF UTAH \\ WASHINGTON STATE UNIVERSITY \\ UNIVERSITY OF WASHINGTON
}

The Supporting Institutions listed above contribute to the cost of publication of this Journal, but they are not owners or publishers and have no responsibility for its content or policies.

Mathematical papers intended for publication in the Pacific Journal of Mathematics should be in typed form or offset-reproduced (not dittoed), double spaced with large margins. Please do not use built up fractions in the text of the manuscript. However, you may use them in the displayed equations. Underline Greek letters in red, German in green, and script in blue. The first paragraph must be capable of being used separately as a synopsis of the entire paper. In particular it should contain no bibliographic references. Please propose a heading for the odd numbered pages of less than 35 characters. Manuscripts, in triplicate, may be sent to any one of the editors. Please classify according to the scheme of Math. Reviews, Index to Vol. 39. Supply name and address of author to whom proofs should be sent. All other communications should be addressed to the managing editor, or Elaine Barth, University of California, Los Angeles, California 90024.

There are page-charges associated with articles appearing in the Pacific Journal of Mathematics. These charges are expected to be paid by the author's University, Government Agency or Company. If the author or authors do not have access to such Institutional support these charges are waived. Single authors will receive 50 free reprints; joint authors will receive a total of 100 free reprints. Additional copies may be obtained at cost in multiples of 50 .

The Pacific Journal of Mathematics is issued monthly as of January 1966. Regular subscription rate: $\$ 132.00$ a year (6 Vol., 12 issues). Special rate: $\$ 66.00$ a year to individual members of supporting institutions.

Subscriptions, orders for numbers issued in the last three calendar years, and changes of address should be sent to Pacific Journal of Mathematics, P.O. Box 969, Carmel Valley, CA 93924, U.S.A. Old back numbers obtainable from Kraus Periodicals Co., Route 100, Millwood, NY 10546.

The Pacific Journal of Mathematics ISSN 0030-8730 is published monthly by the Pacific Journal of Mathematics at P.O. Box 969, Carmel Valley, CA 93924. Application to mail at Second-class postage rates is pending at Carmel Valley, California, and additional mailing offices. Postmaster: Send address changes to Pacific Journal of Mathematics, P. O. Box 969, Carmel Valley, CA 93924.

PUBLISHED BY PACIFIC JOURNAL OF MATHEMATICS, A NON-PROFIT CORPORATION

Copyright $(1983$ by Pacific Journal of Mathematics 


\section{Pacific Journal of Mathematics}

Vol. 108, No. $2 \quad$ April, 1983

Enrique Atencia and Francisco Javier Martin-Reyes, The maximal ergodic Hilbert transform with weights $\ldots \ldots \ldots \ldots \ldots \ldots \ldots \ldots \ldots . \ldots 257$

Bruce Blackadar, The regular representation of local affine motion

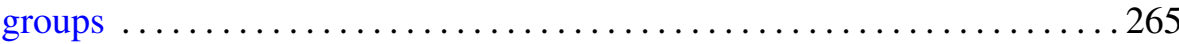

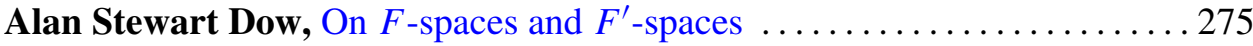

Yoshifumi Kato, On the vector fields on an algebraic homogeneous space . . 285

Dmitry Khavinson, Factorization theorems for different classes of analytic functions in multiply connected domains $\ldots \ldots \ldots \ldots \ldots \ldots \ldots \ldots 295$

Wei-Eihn Kuan, A note on primary powers of a prime ideal . . . . . . . . 319

Benjamin Michael Mann and Edward Yarnell Miller, Characteristic classes for spherical fibrations with fibre-preserving free group

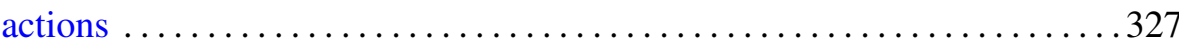

Steven Alan Pax, Appropriate cross-sectionally simple four-cells are flat . . . 379 R. K. Rai, On orthogonal completion of reduced rings ................ 385

V. Sree Hari Rao, On random solutions of Volterra-Fredholm integral

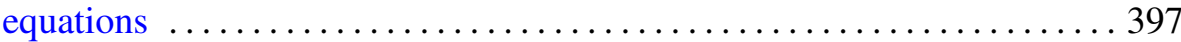

Takeyoshi Satō, Integral comparison theorems for relative Hardy spaces of solutions of the equations $\Delta u=P u$ on a Riemann surface $\ldots \ldots \ldots . .407$

Paul Sydney Selick, A reformulation of the Arf invariant one $\bmod p$ problem and applications to atomic spaces

Roelof Jacobus Stroeker, Reduction of elliptic curves over imaginary quadratic number fields

Jacob Towber, Natural transformations of tensor-products of representation-functors. I. Combinatorial preliminaries

James Chin-Sze Wong and Abdolhamid Riazi, Characterisations of amenable locally compact semigroups 